\title{
Drratum
}

\section{Clinicopathologic Differentiation of Atrophy of the Pancreatic Body and Tail Aplasia}

Koichi Suda, Yoshiro Matsumoto, Hideki Fujii, Kazuo Miura, and Bunsei Nobukawa. Int J Pancreatol 1998; 24: 227-235.

In case 1 described in this article, the authors identified erroneously a dorsal pancreatic artery arising anomalously from the superior mesenteric artery (Fig. 2 and pp. 229,230). In fact, this artery is the arc of Bühler, which represents a primordial vessel owing to the persistence of the embryological ventral anastomotic artery producing a direct connection between the celiac and superior mesenteric arteries. 